\title{
Comunicación estratégica transnacional en Twitter para las elecciones al Parlamento Europeo de 2019
}

\author{
Nazioz gaindiko komunikazio estrategikoa Twitterren \\ $2019 k o$ Europako Parlamenturako hauteskundeetarako \\ Transnational strategic communication on Twitter \\ for the 2019 European Parliament elections
}

\author{
Rubén Rivas-de-Roca \\ Universidad de Sevilla
}

\begin{abstract}
RESUMEN: La Unión Europea sufre de una brecha comunicativa con los ciudadanos, que entronca con la falta de «domesticación» y «politización» de los asuntos comunitarios. Una de las medidas para resolver este problema estructural ha consistido en desarrollar candidatos trasnacionales ("Spitzenkandidaten»). Por ello, este artículo analiza la comunicación estratégica implementada en Twitter por estos candidatos en las elecciones al Parlamento Europeo de 2019. Se aplica un análisis de contenido que estudia sus enfoques estratégicos. Como resultados, se concluye que existe homogeneidad en los elementos utilizados, sin lograr europeizar la agenda. Solo se observan diferencias significativas según el eje europeísmo/euroescepticismo.
\end{abstract}

PALABRAS CLAVE: Comunicación política; estrategia electoral; Twitter; Unión Europea; Spitzenkandidaten.

ABSTRACT: The European Union suffers from a "communications gap» with citizens, which overlaps to the lack of «domestication» and «politicization» of EU affairs. One of the measures to solve this structural problem has been to develop transnational candidates ("Spitzenkandidaten»). To this end, this article analyzes the strategic communication implemented on Twitter by these candidates in the 2019 European Parliament elections. A content analysis is applied to study their strategic approaches. As a result, it is concluded that there is homogeneity in the elements used, without achieving to Europeanize the agenda. Significant differences only come up in the Europeanism / Euroscepticism axis.

KEYWORDS: Political communication; electoral strategy; Twitter; European Union; Spitzenkandidaten.

\footnotetext{
* Correspondencia a / Corresponding author: Rubén Rivas-de-Roca. Departamento Periodismo II. Facultad de Comunicación. Universidad de Sevilla. Avda. Américo Vespucio, s/n (41092 Sevilla) - rrivasderoca@us.es - https://orcid.org/0000-0001-5842-4533

Cómo citar / How to cite: Rivas-de-Roca, Rubén (2020). "Comunicación estratégica transnacional en Twitter para las elecciones al Parlamento Europeo de 2019", Zer, 25(48), 65-83. (https://doi.org/10.1387/zer.21214).

Recibido: 01 noviembre, 2019; aceptado: 06 marzo, 2020.

ISSN 1137-1102 - eISSN 1989-631X / (c) 2020 UPV/EHU

(c) Csta obra está bajo una licencia
} 


\section{Introducción}

La Unión Europea constituye un sistema político de gran influencia en la vida de los más de 500 millones de habitantes residentes en sus Estados miembros, debido a su capacidad legislativa, ejecutiva y judicial. El vínculo democrático con la ciudadanía se produce mediante las elecciones al Parlamento Europeo, que cada cinco años suponen la elección directa de los representantes en esta institución comunitaria. Sin embargo, la UE ha sido tradicionalmente considerada como un ente muy alejado por buena parte de la población, hecho motivado por la falta de interacción directa con las instituciones europeas (Majone, 2005) o por la pervivencia del enfoque periodístico nacional a la hora de informar de estos asuntos (Lloyd y Marconi, 2014).

A la situación de lejanía, se suma en los últimos años una creciente desafección política con el proyecto europeo (Boix Palop y López García, 2014), manifestada en la emergencia de partidos populistas euroescépticos a lo largo del continente. Este auge ya tuvo consecuencias electorales en los comicios europeos de 2014. Hay que tener en cuenta que el voto en las elecciones al Parlamento Europeo suele ser ejercido en clave nacional (Berganza-Conde, 2008; Nulty et al., 2016), funcionando muchas veces como un castigo a las políticas estatales (Hobolt y De Vries, 2014).

A pesar de la distancia ciudadana - la participación en las elecciones europeas se situó en un 43\% en el conjunto de la Unión, tanto en 2009 como 2014, con países por debajo del 25\%, como Polonia y Eslovaquia- y de las críticas que pueda recibir, la UE goza de un importante peso político. Desde sus inicios, y especialmente a partir de la década de 1990 (Andrino San Cristóbal, 2014), la UE ha realizado esfuerzos por comunicar mejor. Estas acciones no han sido estrictamente comunicativas, sino también institucionales. Una de ellas es el llamado sistema de «Spitzenkandidaten» —implantado oficiosamente en 2014-, por el cual los partidos políticos presentan un cabeza de lista que se comprometen a convertir en presidente de la Comisión Europea.

Este sistema trata de mejorar la trazabilidad democrática del proceso electoral europeo, lo que puede haber contribuido a que las elecciones de 2019 cosecharan el primer incremento de la participación en dos décadas, alcanzando el 50\%. No obstante, la promesa de investir a alguno de los candidatos transnacionales por parte de los partidos no tuvo efectos en el Consejo Europeo, que designó a la política alemana Ursula Von der Leyen para presidir la Comisión, a pesar de no haberse presentado a los comicios. En cualquier caso, la justificación de esta investigación radica en que se experimenta actualmente un momento de crisis del proyecto comunitario, en el que resulta de interés conocer qué prácticas adoptan los líderes europeos en una red social de trascendencia política como Twitter. 
Las elecciones de 2019 supusieron la segunda ocasión en que se utilizó el sistema de "Spitzenkandidaten», una consolidación a priori del modelo en la que la experiencia acumulada debería significar la puesta en marcha de mejores estrategias de comunicación. Los objetivos de este mecanismo pasan por elevar la participación y lograr una mayor legitimación ciudadana de la Unión Europea (Aixalà i Blanch, 2014), construyendo auténticos líderes europeos.

Este artículo trata de conocer la actuación en Twitter de los candidatos a presidir la Unión Europea, en lo relativo a la presencia de enfoques estratégicos que busquen la consecución de votos y la interacción que generan en la ciudadanía. Resulta de interés discernir hasta qué punto los encuadres basados en la estrategia se han incorporado al debate electoral europeo, algo que se puede medir a través de las cuentas oficiales de Twitter de estos cabezas de lista, en tanto en cuanto esta red social constituye la mayor herramienta política digital de nuestro tiempo (Campos-Domínguez, 2017).

Las elecciones al Parlamento Europeo corren siempre el peligro de verse intoxicadas por la política nacional, algo aún más probable en 2019 en ciertos países por la coincidencia en fechas con diversos comicios de índole estatal. Por ello, este estudio aplica una metodología que intenta no centrarse exclusivamente en la fase de contienda electoral, tratando de observar la construcción de los líderes europeos en Twitter a lo largo de un período determinado. Ese es el objeto de la investigación, respondiendo a la cuestión de si existen líderes políticos europeos definidos por su comunicación estratégica y, en particular, si los «Spitzenkandidaten» actúan como tales en esa red social.

\section{Antecedentes}

\subsection{La COMUnicación política de La Unión Europea}

La Unión Europea constituye una comunidad de derecho singular, que ha desarrollado un sistema político, dotado de poder legislativo (Parlamento Europeo y Consejo de la UE), ejecutivo (Comisión Europea) y judicial (Tribunal de Justicia de la UE). Por sistema político se alude al conjunto de instituciones que rigen la vida gubernativa de un país (Mazzoleni, 2010), que se ajustaría en este caso a una organización internacional.

Desde los inicios de las Comunidades Europeas en la década de 1950, uno de los objetivos de la UE pasa por darse a conocer a los ciudadanos de los diferentes Estados que la conforman (Tuñón, 2017). Para ello ha empleado una creciente política de comunicación institucional, pasando del modesto servicio de prensa inicial a la actual Dirección General de Comunicación de la Comisión Europea. Los objetivos 
comunitarios en materia comunicativa se establecieron en la Declaración de Laeken de 2001 sobre el futuro de la Unión Europea, que fijaba como misión principal la promoción de una mayor proximidad con el proyecto europeo. Esta declaración se vio complementada en 2006 por el Libro Blanco sobre la Política de Comunicación Europea.

Aunque la UE demuestra una preocupación notable sobre cómo comunicar sus políticas, presente según algunos autores desde la misma concepción del proyecto europeo por Schuman (Andrino San Cristóbal, 2014), hay un consenso académico de dilatada trayectoria en señalar que la Unión no comunica bien (Moravcsik, 2002), transmitiendo una imagen alejada vinculada a la diplomacia (Van Dalen, Albaek y De Vreese, 2011). La literatura sobre integración europea ha apuntado tradicionalmente a la existencia de una brecha comunicativa (communications gap), que impide un diálogo fluido entre las instituciones comunitarias y los ciudadanos (Moravcsik, 2002).

La hipótesis del déficit comunicativo se encuentra muy asentada en los estudios europeos. Las investigaciones sobre este tema se han interesado por la comunicación pública que desarrollan las instituciones de la UE, así como por el papel que unos medios europeizados podrían desempeñar en una supuesta Esfera Pública Europea (Risse, 2010). Esta hipótesis hace hincapié en la mediación, por lo que, de acuerdo a su punto de vista, la comunicación política en la Unión sería un éxito en caso de disponer de unas estrategias más efectivas, que se combinaran con una mayor profesionalidad de los periodistas que cubren asuntos comunitarios.

El fracaso de la Constitución Europea en 2005, rechazada en referéndum en Francia y Países Bajos, ha traído consigo una nueva corriente empírica, más centrada en la dimensión estructural y cultural de la débil comunicación política europea (Schulz-Forberg y Strath, 2010). Un antecedente de este planteamiento fue realizado por Schlesinger (1999), que apuntaba que la UE no era importante en la prensa del continente porque los medios seguían ligados a sistemas políticos nacionales.

Una de las principales aportaciones en el seno de la corriente emergente estructural ha sido la de Seoane Pérez (2013). Este autor explica que las disfunciones trascienden a la comunicación, encontrándose motivadas por un doble problema de «domesticación»y «politización» de los asuntos europeos. La «domesticación»consiste en la falta de identificación de los ciudadanos con la política europea por considerarla alejada de sus preocupaciones cotidianas, mientras que la "politización» refiere a la ausencia de debate político izquierda-derecha en el mundo comunitario, que entorpece la comprensión de los fenómenos políticos europeos.

La conclusión de Seoane Pérez (2013) es que el déficit de la comunicación política europea se debe a la forma en que la UE ha sido construida y gobernada — dos razones estructurales-, y también al tipo de comunidad que ha generado, en lo que 
supone una explicación cultural. Esto imbrica con la doctrina clásica de democracia de Schmitt (1932), que sostiene que este sistema se basa en la vinculación entre gobernantes y gobernados. Para Schmitt (1932), esta conexión requiere de identidad y conflicto, elementos que no se habrían experimentado en la Unión Europea.

En resumen, la literatura sobre la comunicación política europea ha trasladado en los últimos años su enfoque desde la mediación hasta el análisis estructural y cultural de este ámbito. De considerar como clave la dimensión comunicativa se traslada el foco hacia el hecho de que la UE incumple buena parte de los requisitos de un sistema político clásico, dado su carácter sui generis. Sin embargo, el plano institucional debe tener también un componente comunicativo, y es ahí donde entra en juego la estrategia (Aalberg, Strömbäck y De Vreese, 2011). Autores como Tuñón, Bouza y Carral (2019) señalan la necesidad de desarrollar una comunicación estratégica efectiva tanto en las instituciones europeas como en sus líderes políticos, para lograr una democracia comunitaria que pueda denominarse como tal.

Sin duda, la perspectiva estructural y cultural en el análisis de la comunicación política europea se ha erigido en una corriente empírica emergente. Buena parte de sus textos más influyentes se han producido en la última década, como los de Schulz-Forberg y Strath (2010) y Van Dalen, Albaek y De Vreese (2011). Estas investigaciones coinciden con las anteriores en configurar un consenso férreo acerca de la existencia de problemas comunicativos en la UE, constituyendo una aproximación asentada ya detectada en la década de 1980. Esta situación subraya además el rol central que una correcta comunicación estratégica puede desempeñar en la consolidación de la democracia europea.

\subsection{LA DIMENSIÓN DIGITAL DEL SISTEMA DE «SPITZENKANDIDATEN»}

Las elecciones al Parlamento Europeo - momento democrático por excelencia de la UE - se enfrentan a los problemas de falta de legitimidad que se vienen recogiendo, pero también a dificultades provocadas por la actual mediatización de la política. Se trata de un contexto de política mediatizada y democracia de audiencias (Lakoff, 2006), en la que se ganan elecciones desde las tribunas mediáticas. Sin embargo, la UE no posee un sistema de medios propio, ni tampoco cuenta con un tratamiento periodístico ad hoc, elementos básicos para el desarrollo de la comunicación mediática (Mazzoleni, 2010).

La propia UE ha demostrado en los últimos años una preocupación por las causas estructurales de las disfunciones de su comunicación política. En las elecciones al Parlamento Europeo de 2014 se instauró por primera vez el sistema de «Spitzenkandidaten». Si bien esta prerrogativa no se encuentra escrita en los Tratados Europeos, las instituciones comunitarias la han impulsado para crear la sensación de que se vota 
a un presidente para Europa, en un intento de hacer frente a la crisis democrática de la UE (Aixalà i Blanch, 2014).

El sistema de «Spitzenkandidaten» significa a priori una politización y personalización de las elecciones al Parlamento Europeo. En cualquier caso, este procedimiento apenas ha sido estudiado todavía en España, como lo atestigua nuestra búsqueda de información y una reciente tesis doctoral sobre la política de información de la UE (Benedicto Solsona, 2016). A nivel europeo, la Eurocámara ha respaldado investigaciones específicas, que señalan una cierta ineficacia de este sistema para aumentar la participación en las elecciones de 2014 (Schmitt, Hobolt y Popa, 2015).

Un factor clave en el fenómeno de personalización política en las democracias avanzadas son las plataformas digitales (Cotarelo, 2010). Internet generó un optimismo académico sobre la posibilidad de construir una esfera pública digital a nivel europeo que contrarrestara la falta de un sistema mediático ad hoc (Thomass, 2011). La UE ha apoyado iniciativas europeístas digitales, pero esto no ha impedido fenómenos como el Brexit, unido al hecho de que la confianza en el proyecto europeo se encuentre en uno de los momentos más bajos de la historia (De Areilza e Imbernón, 2018). Los estudios empíricos más recientes, como el de Ramos-Serrano, Fernández Gómez y Pineda (2018), reflejan una pervivencia de los enfoques nacionales en la discusión pública comunitaria.

Twitter es la herramienta digital que mejor se ha adaptado al campo de la comunicación política (Parmelee y Bichard, 2012). Por sus características intrínsecas, como su inmediatez, facilidad de uso o propensión a la viralidad, esta red social de microblogging se ajusta a la perfección al nuevo discurso público fragmentario mediado por las tecnologías digitales (Barber, 2004: 38). Twitter se erige así en un instrumento ideal para que los líderes políticos puedan determinar la agenda mediática, práctica observable en el caso de Donald Trump en Estados Unidos.

Twitter desempeña una serie de funciones en la comunicación política. La más habitual es la difusión de declaraciones políticas, así como informar sobre eventos en caso de encontrarse en campaña electoral (Jackson y Lilleker, 2011). De esta manera predomina una función de autorreferencialidad (Ignacio-Criado, Martínez-Fuentes y Silván, 2013), que vertebra una personalización de la política. Otro rol atribuido a Twitter es su capacidad de movilizar el voto y la participación (Gainous y Wagner, 2014), lo cual es de extraordinaria importancia para los cabezas de lista europeos. También se suele vincular a Twitter con la interacción con el público. Sin embargo, las evidencias empíricas señalan que no se está produciendo un grado de intercambio significativo (Nulty et al., 2016).

La literatura sobre comunicación política en Twitter recoge que este medio digital no fomenta una elevada fragmentación de la agenda (Segado-Boj, Díaz-Campo 
y Lloves, 2016; Alonso-Muñoz y Casero-Ripollés, 2018), es decir, de los temas tratados por los líderes políticos. Hay investigaciones (Enli, 2017) que han demostrado la capacidad que las redes sociales tienen de construir agenda pública para aquellos actores que están fuera de ella, como las fuerzas populistas, pero también los partidos europeos transnacionales, normalmente olvidados por los medios de comunicación (Lloyd y Marconi, 2014).

Aunque no se refieren a las elecciones al Parlamento Europeo, sí que se han producido otras investigaciones que han analizado en Twitter fenómenos políticos europeos, como los líderes populistas. Un estudio de Casero-Ripollés, Sintes-Olivella y Franch (2017) revela que su agenda tiene un enfoque más temático, y por ende menos estratégico, que el de los políticos tradicionales. El hecho de que el debate en redes sociales sobre las elecciones al Parlamento Europeo se produzca en términos nacionales (Ramos-Serrano, Fernández Gómez y Pineda, 2018) evidencia que, a pesar de que las nuevas medidas adoptadas por las instituciones europeas en materia comunicativa hayan sido valoradas como esfuerzos significativos (Bruter, 2005), estos comicios no poseen una repercusión efectiva en la consecución de una mayor conexión con la ciudadanía.

\section{Metodología}

El objetivo de esta investigación es conocer el uso estratégico que realizan de Twitter los cabezas de lista transnacionales («Spitzenkandidaten») para las elecciones al Parlamento Europeo de 2019, permitiendo profundizar sobre el buen funcionamiento de este mecanismo, en el sentido de mejorar la legitimidad de la UE. Se reconocen unos objetivos específicos:

O1. El estudio de la agenda estratégica que estos candidatos exponen en sus perfiles oficiales de Twitter.

O2. Comparación de las estrategias comunicativas empleadas, en cuanto al uso de una agenda temática o estratégica para la consecución de votos, que entronque con uno de los polos del eje cosmopolitismo/nacionalismo. La visión cosmopolita hace referencia a valores postindustriales de pluralismo y tolerancia.

O3. Análisis de la atención que genera la acción de los cabezas de lista en Twitter, formulada a través de retweets y favoritos.

Partiendo de la revisión de la literatura efectuada, se establecen una serie de hipótesis:

H1. Existe una diferencia estratégica entre los partidos del eje izquierda/derecha, así como entre los del eje europeísmo/euroescepticismo. 
H2. El enfoque estratégico, expresado sobre el grado de europeísmo, acapara un número mayoritario de mensajes, siendo menor en las fuerzas euroescépticas.

H3. Las cuestiones nacionales gozan de una importante presencia en los tweets de los candidatos, por lo que el sistema de «Spitzenkandidaten» no habría conseguido su objetivo de europeizar la estrategia europea.

H4. La personalización de los mensajes es baja, en consonancia con la cultura política tradicional europea contraria a la individualización de las campañas.

Para lograr las metas propuestas se emplea el análisis de Twitter (Fernández Crespo, 2014), basado en principios de contenido cuantitativo, cuya adecuación al ámbito de la comunicación ha quedado refrendada en diversas investigaciones (Krippendorff, 1990). Su utilidad radica en que permite entender los componentes que forman parte del mensaje comunicativo (Igartua, 2006), aplicado aquí a la singularidad de las redes sociales. Siguiendo este enfoque, se analizan los perfiles de los «Spitzenkandidaten» proclamados:

- Manfred Weber (Partido Popular Europeo-PPE, Alemania), @ManfredWeber (cuenta única en inglés y alemán).

- Frans Timmermans (Partido Socialista Europeo-PES, Países Bajos), @TimmermansEU (cuenta única en inglés).

- Jan Zahradil (Alianza de los Conservadores y Reformistas Europeos-ACRE, República Checa), @Zahradiljan (cuenta única en inglés y checo).

- Bas Eickhout (Partido Verde Europeo-GREENS, Países Bajos), @BasEickhout (cuenta única en inglés y neerlandés).

- Ska Keller (Partido Verde Europeo-GREENS, Alemania), @SkaKeller (cuenta única en inglés y alemán).

— Nico Cué (Partido de la Izquierda Europa-IE, Bélgica), @AvecNico (cuenta única en inglés y francés).

Como se puede observar, el Partido Verde Europeo (GREENS) presenta dos candidatos, en un intento de mantener la lógica coral de la organización. El Partido de la Izquierda Europea (IE) también posee dos aspirantes - Nico Cué y la eslovena Violeta Tomič - si bien Tomič tiene una cuenta de Twitter escasamente activa (menos de un tweet al mes), de ahí que no haya sido incluida en este trabajo.

La investigación solo analiza a personalidades que hayan sido seleccionadas como candidatos de lista transnacionales. Esto excluye al Partido de la Alianza de los Liberales y Demócratas por Europa (ALDE), que ha decidido abandonar en esta ocasión el procedimiento de «Spitzenkandidaten» por no estar de acuerdo con su funcionamiento, al limitar la capacidad de decisión del Consejo Europeo.

El conjunto de esta investigación trata de medir la construcción del perfil de los candidatos. Por ello, como período de seguimiento se selecciona una franja más amplia 
que la campaña electoral oficial, analizando los mensajes durante un período de tres meses, del 22 de febrero al 22 de mayo de 2019, dado que el 23 comienzan las elecciones al Parlamento Europeo, que se celebran durante cuatro días en los diversos países europeos. Se establece el comienzo tres meses antes porque es el momento en el que el Parlamento Europeo hace públicas sus primeras proyecciones de escaños, que se prolongan hasta los comicios, en lo que entraña el pistoletazo de salida a la precampaña.

El estudio tiene en cuenta los tweets propios y las respuestas de los cabezas de lista europeos, pero no los retweets, puesto que contienen información publicada por otros y no nos permiten conocer las agendas y estrategias de cada candidato (Larsson e Ihlen, 2015). La recogida de datos se lleva a cabo mediante Twitonomy, siendo analizada posteriormente por software estadístico. Se genera una muestra de 1.444 tweets, repartidos de la siguiente forma entre los candidatos: 294 mensajes de Weber (PPE), 291 de Timmermans (PES), 371 de Zahradil (ACRE), 125 de Keller (GREENS), 241 de Eickhout (GREENS) y 122 de Cué (IE).

TABLA 1

Categorías empleadas para el estudio cuantitativo de la comunicación estratégica en Twitter

\begin{tabular}{c|l|l}
\hline \multirow{2}{*}{$\begin{array}{c}\text { Enfoque } \\
\text { temático }\end{array}$} & $\begin{array}{l}\text { Temas } \\
\text { Todos los mensajes con } \\
\text { naturaleza programática }\end{array}$ & $\begin{array}{l}\text { Tweets sobre cuestiones temáticas, entre los que destacan el } \\
\text { Brexit, la economía, la seguridad, la inmigración o el me- } \\
\text { dioambiente. }\end{array}$ \\
\hline \multirow{5}{*}{$\begin{array}{c}\text { Enfoque } \\
\text { estratégico }\end{array}$} & $\begin{array}{l}\text { Estrategia y pactos de } \\
\text { gobierno }\end{array}$ & $\begin{array}{l}\text { Tweets que hacen referencia a posibles pactos post-electo- } \\
\text { rales en la conformación de la Comisión Europea. Se sitúan } \\
\text { aquí las menciones a encuestas. }\end{array}$ \\
\cline { 2 - 3 } & Enfoque de conflicto & $\begin{array}{l}\text { Tweets que adoptan una posición de agresividad (buenos/ } \\
\text { malos) hacia el contrincante político. }\end{array}$ \\
\cline { 2 - 4 } & $\begin{array}{l}\text { Organización de } \\
\text { eventos políticos }\end{array}$ & $\begin{array}{l}\text { Tweets sobre aspectos de la vida personal de los cabezas de } \\
\text { lista europeos. }\end{array}$ \\
\cline { 2 - 3 } & $\begin{array}{l}\text { Relación con los medios acerca de eventos políticos, como debates electorales } \\
\text { de comunicación }\end{array}$ & $\begin{array}{l}\text { Tweets relacionados con los medios de comunicación, como } \\
\text { puede ser la aparición del candidato en uno de ellos o la exis- } \\
\text { tencia de discrepancias con una publicación periodística. }\end{array}$ \\
\hline \multirow{2}{*}{ Otros } & Tweets que no se pueden \\
\hline
\end{tabular}

Fuente: elaboración propia.

Se ha desarrollado una ficha de análisis (tabla 1) con categorías excluyentes para estudiar los tweets analizados. Esta tabla cuenta con una variable aglutinadora para 
la agenda temática y con categorías específicas dedicadas a la estrategia, en cuanto constituyen el objeto de la investigación. Para estas últimas se sigue la definición de Aalberg, Strömback y De Vreese (2011), ubicando bajo la denominación de game frame los enfoques estratégicos y de juego electoral, es decir, todos aquellos que hacen referencia a mensajes que buscan la obtención de votos.

\section{Resultados}

\subsection{La estrategia COMunicativa «Spitzenkandidaten» en Twitter}

El análisis de la agenda formulada por los seis «Spitzenkandidaten» arroja evidencias empíricas de interés. Su contenido se revela mucho más estratégico que temático (tabla 2), es decir, más orientado a la disputa electoral que a la difusión de propuestas (Berganza-Conde, 2008). Todos los candidatos publican porcentualmente más tweets dotados de enfoques estratégicos que de ofertas programáticas: 54,3\% a $38,6 \%$ en Weber, $48,9 \%$ a $43,9 \%$ en Timmermans, $62,3 \%$ a $18,1 \%$ en Zahradil, $45,6 \%$ a $40 \%$ en Keller, $47,8 \%$ a $31,1 \%$ en Eickhout y $66,4 \%$ a $27.1 \%$ en Cué.

TABLA 2

Distribución de los mensajes según las variables de enfoque temático y estratégico $(\%)$

\begin{tabular}{|c|c|c|c|c|c|c|c|}
\hline & & $\begin{array}{c}\text { Manfred } \\
\text { Weber }\end{array}$ & $\begin{array}{c}\text { Frans } \\
\text { Timmer. }\end{array}$ & $\underset{\text { Zahradil }}{\text { Jan }}$ & $\begin{array}{c}\text { Ska } \\
\text { Keller }\end{array}$ & $\begin{array}{c}\text { Bas } \\
\text { Eickhout }\end{array}$ & $\begin{array}{l}\text { Nico } \\
\text { Cué }\end{array}$ \\
\hline \multicolumn{2}{|c|}{ Enfoque temático } & 38,6 & 43,9 & 18,1 & 40,0 & 31,1 & 27,1 \\
\hline \multirow{6}{*}{$\begin{array}{l}\text { Enfoque } \\
\text { estratégico }\end{array}$} & $\begin{array}{l}\text { Estrategia y pactos } \\
\text { de gobierno }\end{array}$ & 7,5 & 7,9 & 15,1 & 4,0 & 4,6 & 3,3 \\
\hline & $\begin{array}{l}\text { Enfoque de } \\
\text { conflicto }\end{array}$ & 10,2 & 12,4 & 27,8 & 11,2 & 13,3 & 13,1 \\
\hline & Temas personales & 2,7 & 4,5 & 2,7 & 1,6 & 5,8 & 17,2 \\
\hline & $\begin{array}{l}\text { Organización de } \\
\text { eventos políticos }\end{array}$ & 26,8 & 21,0 & 10,2 & 25,6 & 15,8 & 27,0 \\
\hline & $\begin{array}{l}\text { Relación con } \\
\text { los medios de } \\
\text { comunicación }\end{array}$ & 7,1 & 3,1 & 6,5 & 3,2 & 8,3 & 5,8 \\
\hline & Total & 54,3 & 48,9 & 62,3 & 45,6 & 47,8 & 66,4 \\
\hline \multicolumn{2}{|l|}{ Otros } & 7,1 & 7,2 & 19,6 & 14,4 & 21,1 & 6,5 \\
\hline \multicolumn{2}{|l|}{ Total } & 100,0 & 100,0 & 100,0 & 100,0 & 100,0 & 100,0 \\
\hline
\end{tabular}

Fuente: datos de la investigación. Elaboración propia. 
El elemento estratégico más referenciado por la mayoría de los candidatos es la organización de eventos políticos. Zahradil (ACRE) supone la excepción, ya que el enfoque de conflicto constituye su estrategia predominante. Zahradil es el único «Spitzenkandidaten» abiertamente euroescéptico, pero no el único crítico con la actual UE. Cué (IE) también se muestra reacio en este sentido. Llama la atención en que son precisamente estos dos candidatos los que más recurren a encuadres estratégicos - 62,3\% en Zahradil y 66,4\% en Cué-, comportamiento que no se puede entender desde el polo ideológico izquierda-derecha.

Otro elemento destacable detectado por nuestra investigación es la elevada presencia del parámetro «otros» en el uso de Twitter de Zahradil (ACRE) y Eickhout (GREENS), así como en menor medida en el caso de Keller (GREENS). En «otros» se han recogido mensajes que no se ajustaban a los parámetros fijados, aludiendo en la mayoría de los casos a interacciones y agradecimientos personales. Este dato resulta digno de mención, puesto que numerosos artículos científicos han indicado que los políticos rara vez emplean las réplicas en esta red social (Campos-Domínguez, 2017). Los candidatos de GREENS y ACRE, desde el euroescepticismo y el ecologismo respectivamente, demuestran cierta preocupación por la interacción con la audiencia.

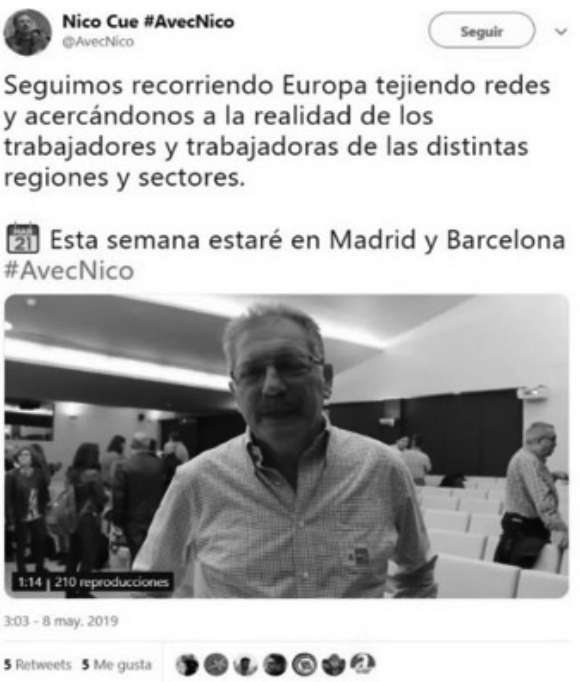

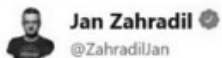

Mr.@TimmermansEU, @PES_PSE \#spitzenkandidat wants to fight "nationalists" and "populists" in \#EP elections. Mr. @ManfredWeber, @EPP \#spitzenkandidat, wants to do the same. And in fact, both their parties will lose, unable to deal with real \#EU problems. @ACREurope \#RetuneTheEU
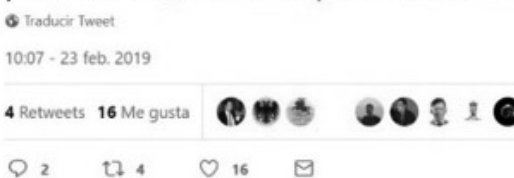

Fuente: Twitter.

\section{IMAGEN 1}

Ejemplos de tweets relativos a organización de eventos (Cué) y enfoque de conflicto (Zahradil)

También genera interés el hecho de que la utilización de enfoques estratégicos propios de las campañas electorales, como la organización de eventos políticos, se 
mantenga constante a lo largo de los tres meses de la investigación, que solo incluyen 15 días de campaña oficial. Este hallazgo en las elecciones europeas concuerda con la idea asentada de campaña permanente en las sociedades postindustriales, de tal forma que los períodos electorales se difuminan y amplían en el tiempo (Mazzoleni, 2010).

En el perfil de Zahradil (ACRE), único candidato euroescéptico, no se observa una práctica propia de los líderes populistas, que consiste en primar una agenda temática de realizaciones concretas (Alonso-Muñoz y Casero-Ripollés, 2018). Hay que valorar que Zahradil pertenece a una coalición europea que acepta de manera crítica la UE como marco institucional, existiendo posturas más reaccionarias en el conjunto de países de la Unión.

A la vista de lo anterior, parece claro que no se puede realizar una traslación exacta entre los partidos nacionales y las fuerzas que se presentan a nivel europeo. No obstante, dentro de la franja temporal acotada se han observado interesantes fenómenos, como el enfoque de conflicto en el euroescepticismo y la preferencia por la organización de eventos en la mayoría de los candidatos. El conjunto de los «Spitzenkandidaten» recurre más a elementos estratégicos que temáticos, sin que se aprecien diferencias en el eje izquierda/derecha. Las divergencias tienden a aparecer en la agenda programática, donde un análisis detallado mostraría la predilección por cierto temas, como la política social, que han tenido los candidatos de izquierda.

\subsection{INTERACCIÓN DE LOS USUARIOS RESPECTO A LA ESTRATEGIA}

El análisis del impacto de la agenda desarrollada en Twitter por los «Spitzenkandidaten» muestra una mayor interacción con los temas programáticos en comparación con los encuadres estratégicos, proceso evaluado a través de la media de retweets y favoritos. El conjunto de mensajes temáticos presenta una media de 23,25 retweets y 69,28 favoritos, frente a 15,59 retweets y 48,89 favoritos de los contenidos estratégicos (tabla 3 ). Por encima del promedio total (21,04 y 64,12, respectivamente) solo se sitúa el marco de estrategia y pactos de gobierno.

Los resultados reflejan que los encuadres que generan un mayor grado de interacción no siempre coinciden con lo más difundido por los «Spitzenkandidaten». A pesar de que la organización de eventos es el frame más aludido, el marco referente a estrategia y pactos de gobierno acapara un número superior de retweets y favoritos. El público demuestra así un interés por conocer la composición del futuro gobierno comunitario, frente a la publicidad de actos políticos propia de la campaña. 
TABLA 3

Media de retweets y favoritos según la temática/agenda de los mensajes de los «Spitzenkandidaten» (en negrita por encima de la media)

\begin{tabular}{lcc}
\hline \multicolumn{1}{c}{ Temática/agenda } & Retweets & Favoritos \\
\hline Subtotal enfoque temático & $\mathbf{2 3 , 2 5}$ & $\mathbf{6 9 , 2 8}$ \\
Estrategia y pactos de gobierno & $\mathbf{2 8 , 7 0}$ & $\mathbf{7 7 , 8 3}$ \\
Enfoque de conflicto & 14,42 & 44,76 \\
Temas personales & 8,62 & 35,05 \\
Organización de eventos políticos & 16,78 & 59,92 \\
Relación con los medios de comunicación & 9,47 & 26,93 \\
Subtotal enfoque estratégico & 15,59 & 48,89 \\
Otros & 19,46 & 73,20 \\
\hline Total & 21,04 & 64,12 \\
\hline
\end{tabular}

Fuente: datos de la investigación. Elaboración propia.

Según los datos de la tabla 3, la audiencia escoge interactuar más con el programa que con los elementos estratégicos. Si bien los contenidos programáticos escasean en los mensajes de los candidatos, son precisamente estas propuestas concretas las que generan una mayor atención por parte de la ciudadanía. Las cuestiones estratégicas se revelan como de menor interés para la audiencia, al menos en lo que a participación en Twitter se refiere. Esto debe obligar a una reflexión sobre la incapacidad de los líderes europeos de llevar a cabo una comunicación persuasiva, que consiga movilizar a los receptores.

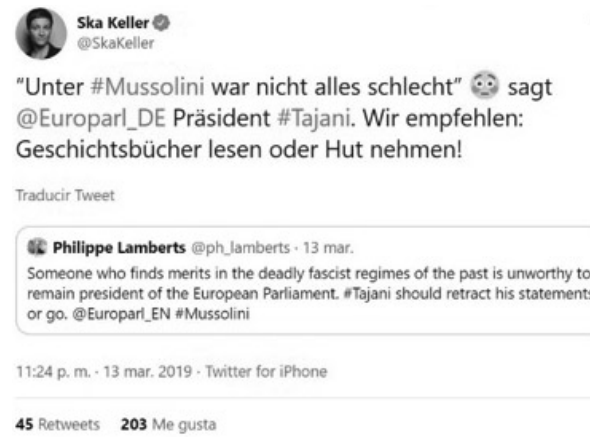

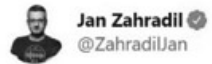

Enhorabuena a @vox_es y a @Santi_ABASCAL por su fuerte entrada en el Parlamento de España.

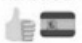

9:08 a. m. $\cdot 29$ abr. $2019 \cdot$ Twitter for iPhone

58 Retweets 403 Me gusta

Fuente: Twitter.

\section{IMAGEN 2}

Tweets estratégicos y de la categoría "otros» que resultan de mayor interés para el público. Enfoque de conflicto (Keller) y «otros» (Zahradil)

Prueba de lo anterior es que un mensaje enmarcado en la categoría «otros» consigue un nivel de interacción mayor que el tweet estratégico que generó más atención, publicado por Keller (GREENS) con un enfoque de conflicto (imagen 2). 
El tweet de «otros» que provoca más implicación se refiere a la felicitación del candidato euroescéptico al partido Vox por su entrada en el Congreso de los Diputados en abril de 2019. Esto denota hasta qué punto las cuestiones nacionales se insertan en la campaña de los líderes transnacionales en las europeas, algo probablemente motivado por el hecho de que esos asuntos son los que más interesan a la ciudadanía. La atención derivada de los elementos estratégicos es escasa, por lo que no se puede hablar de un éxito comunicativo del sistema de «Spitzenkandidaten».

Se ha medido también hasta qué punto los intereses del público difieren de los planteados por los «Spitzenkandidaten» en nuestra investigación. Para evaluar la fuerza y la dirección de la asociación, se aplica el coeficiente de correlación de Pearson $(r=0,126)$, que presenta unos valores bajos entre el número de retweets y favoritos conseguidos por cada mensaje y la cifra de mensajes publicados. Como se puede observar, se aproxima al valor 0 , que indica la inexistencia de relación entre las variables. En nuestro caso hay un cierto vínculo, ya que el valor 0,126 se califica como correlación positiva muy baja, al hallarse entre 0,01 y 0,19 . Esto evidencia que la atención de los usuarios no tiene que ver de forma significativa con el número de mensajes difundidos.

La cifra de tweets no es relevante para una mayor o menor interacción de los usuarios con los líderes políticos. De acuerdo a nuestra investigación, para ellos resulta más importante que estos mensajes refieran a cuestiones temáticas frente a elementos estratégicos, a la vez que se refleja una cierta indiferencia hacia los asuntos estrictamente europeos. Los temas nacionales, incluso anecdóticos como la felicitación a un aliado político, aglutinan más retweets y favoritos.

\section{Discusión y conclusiones}

Como discusión de este artículo, cabe señalar que el sistema de «Spitzenkandidaten» tiene un indudable componente estructural que se enmarca en una nueva clase de propuestas para hacer frente a la secular brecha comunicativa de las instituciones europeas (Seoane Pérez, 2013). Su pretensión es europeizar el debate público (Aixalà i Blanch, 2014), algo que encaja con las preferencias de los receptores de acuerdo a esta investigación. Los ciudadanos en Twitter prefieren interactuar más con los temas programáticos comunitarios que con los encuadres estratégicos. Sin embargo, los «Spitzenkandidaten» priorizan esta dimensión estratégica, especialmente a través de la organización de eventos políticos, un elemento que no consigue conectar con la audiencia de acuerdo a los datos de retweets y favoritos.

El eje europeísmo/euroescepticismo funciona como marco explicativo de la acción comunicativa de los candidatos analizados, lo que se encuentra relacionado con un choque de valores culturales entre cosmopolitismo y nacionalismo que permea la actividad política actual en Estados Unidos y Europa (Norris e Inglehart, 
2018). Más allá de esta disputa, se observa falta de conflicto entre los partidos pertenecientes a ideologías de izquierda y derecha («politización»), con un comportamiento muy similar entre los «Spitzenkandidaten» de ambos polos. Solo el líder euroescéptico se desmarca de esta tendencia, un hallazgo que debería ser objeto de investigaciones futuras.

Asimismo, se percibe una ausencia de identificación con los temas europeos por parte de los receptores en Twitter. Una prueba es que los asuntos que generan un mayor volumen de interacciones son aquellos que se vinculan con el ámbito nacional. Los Estados miembros siguen revelándose como fundamentales para entender la comunicación de la UE (Schmitt, Hobolt y Popa, 2015). Hay que tener en cuenta que, tras las elecciones comunitarias, el Consejo Europeo optó por una candidata que no se había presentado a los comicios, lo que denota que el sistema transnacional no contaba con el apoyo de todas las instituciones. Por tanto, a pesar de este intento de politización, el debate europeo mantiene su tendencia clásica al consenso y la falta de conflicto (Majone, 2005).

En cualquier caso, los resultados de esta investigación aportan una serie de conclusiones de interés sobre la comunicación en Twitter de los «Spitzenkandidaten» para las elecciones al Parlamento Europeo de 2019, que se ordenan tratando de verificar las hipótesis del estudio, a la vez que se da respuesta a los objetivos planteados:

1. En los perfiles de Twitter analizados se produce una diferencia estratégica entre los partidos según el eje europeísmo/euroescepticismo, pero no de acuerdo al eje izquierda/derecha, lo que valida solo en una parte nuestra primera hipótesis que afirmaba la existencia de esta doble distinción. La dimensión con mayor poder explicativo es la de europeísmo/euroescepticismo, dado que el euroescéptico Zahradil (ACRE) presenta una comunicación distinta al resto de candidatos, con relevancia del encuadre estratégico de conflicto, proceder propio de las fuerzas populistas.

2. La segunda de nuestra hipótesis, referente a que el enfoque estratégico acaparaba un número mayoritario de mensajes, siendo menor en las fuerzas euroescépticas, se verifica solo en su primera parte. Los «Spitzenkandidaten» han empleado de forma predominante durante el período de la investigación los encuadres estratégicos. Sin embargo, los datos recabados invalidan la afirmación de que la estrategia sea menor en las fuerzas euroescépticas. Todo lo contrario: a nivel de "Spitzenkandidaten", el único líder euroescéptico ha sido el que más ha utilizado la estrategia. La preferencia temática de los líderes populistas por la tematización, recogida por la literatura, no ha sido localizada.

3. Se valida también la tercera hipótesis, que aludía a que las cuestiones nacionales poseen una importante presencia en los tweets de los candidatos, reflejo de que el sistema de «Spitzenkandidaten» no ha conseguido dar lu- 
gar a una agenda política comunitaria. Se aprecian escasas referencias a Europa como sujeto. La mayor muestra de ello es que los «Spitzenkandidaten» han apostado por una agenda estratégica que prima la difusión de eventos por encima de marcos que podrían causar una europeización de la discusión pública, como la estrategia y pactos electorales o la relación con los medios de comunicación, dando a conocer noticias al respecto.

4. La última de las hipótesis de nuestra investigación, que sostenía que la personalización de los mensajes era baja, predominando una función de difusión de las propuestas políticas, se valida solo en su primera parte. Como es habitual en los países europeos, el encuadre de temas personales es citado limitadamente. Solo recurren a él de forma un poco más frecuente desde la izquierda, lo que resulta sorprendente atendiendo a su tradición colectiva. Por tanto, se verifica la baja personalización de la política comunitaria, pero esto no significa que se prioricen las propuestas temáticas. Como se ha visto, los «Spitzenkandidaten» conceden más espacio a los componentes estratégicos en comparación con el ámbito programático.

En resumen, el sistema de «Spitzenkandidaten» ha supuesto un intento de politizar la UE para conectarla con la ciudadanía, tratando de afrontar la crítica tradicional de déficit comunicativo atribuida a las instituciones europeas. En los tres meses previos a las elecciones de 2019, la comunicación de los «Spitzenkandidaten» se ha caracterizado por el empleo de enfoques estratégicos, si bien limitados a cuestiones muy tangenciales como la difusión de eventos. El hecho de que los cabezas de lista no hayan actuado como verdaderos líderes comunitarios en Twitter demuestra que Europa sigue sin disponer de una comunicación estratégica efectiva a nivel transnacional. En consecuencia, la ciudadanía presenta una preferencia en su interacción por las propuestas programáticas.

El debate de temas concretos en el plano europeo resulta sin duda de interés, pero son los elementos estratégicos los que pueden engarzar con una mayor implicación de la ciudadanía, de ahí que sean de tanta relevancia para el proyecto comunitario. Además, los componentes estratégicos se establecen como característicos de la comunicación política moderna, en la que se debería situar la acción en Twitter de los «Spitzenkandidaten».

El eje de europeísmo/euroescepticismo, que entronca con la disputa entre cosmopolitismo y nacionalismo que marca la UE de nuestro tiempo, se antoja muy relevante explicativamente, puesto que el actor analizado poco favorable a la Unión ha tenido un comportamiento diferencial en balance con el resto, en una prueba de las distintas estrategias comunicativas que adoptaron los «Spitzenkandidaten» para las elecciones al Parlamento Europeo de 2019. Unas estrategias que no han logrado el grado de europeización deseado con la formulación del nuevo sistema de candidatos y que debe movilizar la reflexión sobre el trabajo realizado, si bien en esos comicios 
se experimentó un aumento de la participación que podría ser evaluado como resultado de la comunicación europea, tanto de los «Spitzenkandidaten» como a nivel oficial por parte de las instituciones comunitarias.

\section{Referencias bibliográficas}

Aalberg, T., Strömback, J. y De Vreese, C.H. (2011). «The framing of politics as strategy and game: A review of concepts, operationalizations and key findings». Journalism, 13(2), 162-178. https://doi.org/10.1177/1464884911427799

Aixalà i Blanch, A. (2014). ¿Un Presidente para Europa? Las elecciones de 2014 como respuesta a la crisis democrática europea. Barcelona: Fundació Catalunya Europa.

Alonso-Muñoz, L. y Casero-Ripollés, A. (2018). «Communication of European populist leaders on Twitter: agenda setting and the "more is less" effect». El profesional de la información, 27 (6), 1193-1202. https://doi.org/10.3145/epi.2018.nov.03

Andrino San Cristóbal, S. (2014). Comunicar sobre Europa, desde Europa y para Europa. La política de comunicación europea entre 1990 y 2010: Euranet, la primera red de radios europeas. Valladolid: Universidad de Valladolid. Secretariado de Publicaciones.

Barber, B. (2004). «Which Technology and Which Democracy?». En H. Jenkins, y D. Thorburn (eds.), Democracy and New Media (pp. 33-48). Cambridge, MA: The M.I.T Press.

Benedicto Solsona, M.A. (2016). Política de información y comunicación de la Unión Europea. Una estrategia insuficiente para cerrar la brecha entre la ciudadanía y la UE. (Tesis doctoral). Dirigida por Félix Maraña Marcos y Juan Luis Manfredi Sánchez. Madrid: Universidad Complutense de Madrid.

Berganza-Conde, M.R. (2008). «Medios de comunicación, «espiral del cinismo»y desconfianza política. Estudio de caso de la cobertura mediática de los comicios electorales europeos». ZER-Revista de estudios de comunicación, 13 (25), 121-139.

Boix Palop, A. y López García, G. (2014). «El significado de las Elecciones Europeas de 2014 en España: giro a la izquierda y hundimiento del bipartidismo». Unión Europea Aranzadi, 7, 69-93.

Bruter, M. (2005). Citizens of Europe? The emergence of a mass European identity. Nueva York: Palgrave Macmillan.

Campos-Domínguez, E. (2017). «Twitter y la comunicación política». El profesional de la información, 26 (5), 785-793. https://doi.org/10.3145/epi.2017.sep.01

Casero-Ripollés, A., Sintes-Olivella, M. y Franch, P. (2017). «The populist political communication style in action: Podemos's issues and functions on Twitter during the 2016 Spanish general election». American Behavioral Scientist, 61 (9), 986-1001. https://doi. org/10.1177/0002764217707624

Cotarelo, R. (2010). La política en la era de internet. Valencia: Tirant lo Blanch.

Ignacio-Criado, J.I., Martínez-Fuentes, G. y Silván, A. (2013). «Twitter en campaña: las elecciones municipales españolas de 2011». RIPS: Revista de Investigaciones Políticas y Sociológicas, 12 (1), 93-113. 
De Areilza, J.M. e Imbernón, A. (2017). «Un guion español para la UE». Política exterior, 31(180), 94-104.

Enli, G. (2017). «Twitter as arena for the authentic outsider: Exploring the social media campaigns of Trump and Clinton in the 2016 US presidential election». European Journal of Communication, 32 (1), 50-61. https://doi.org/10.1177/0267323116682802

Fernández Crespo, M. (2014). «Metodología para el análisis de las redes sociales». En R. Cotarelo, y J.A. Olmeda (eds.), La democracia del siglo XXI. Política, medios de comunicación, internet $y$ redes sociales (pp. 383-408). Madrid: Centro de Estudios Políticos y Constitucionales.

Gainous, J. y Wagner, K.M. (2014). Tweeting to power: The social media revolution in American politics. Oxford: Oxford University Press.

Hobolt, S.B. y De Vries, C. (2016). «Turning against the Union? The impact of the crisis on the Eurosceptic vote in the 2014 European Parliament elections». Electoral Studies, 44, 504-514. https://doi.org/10.1016/j.electstud.2016.05.006

Igartua, J.J. (2006). Métodos cuantitativos de investigación en comunicación. Barcelona: Bosch.

Jackson, N. y Lilleker, D. (2011). «Microblogging, constituency service and impression management: UK MPs and the use of Twitter». The Journal of Legislative Studies, 17(1), 86-105. https://doi.org/10.1080/13572334.2011.545181

Krippendorff, K. (1990). Metodología de análisis de contenido. Teoría y práctica. Barcelona: Paidós Comunicación.

Lakoff, G. (2006). No pienses en un elefante. Madrid: Editorial Complutense.

Larsson, A.O. e Ihlen, O. (2015). «Birds of a feather flock together? Party leaders on Twitter during the 2013 Norwegian elections». European Journal of Communication, 30 (6), 666681. https://doi.org/10.1177/0267323115595525

Lloyd, J. y Marconi, C. (2014). Reporting the EU: news, media and the European institutions. Londres: I.B. Tauris.

Majone, G. (2005). Dilemmas of European integration: The ambiguities and pitfalls of integration by stealth. Oxford y Nueva York: Oxford University Press.

Mazzoleni, G. (2010). La comunicación política. Madrid: Alianza.

Moravcsik, A. (2002). «In defence of the "democratic deficit": Reassessing legitimacy in the European Union». Journal of Common Market Studies, 40(4), 603-624.

Norris, P. e Inglehart, R. (2019). Cultural Backlash: Trump, Brexit, and Authoritarian Populism. Cambridge: Cambridge University Press.

Nulty, P., Theocharis, Y., Popa, S.A., Parnet, O. y Benoit, K. (2016). «Social Media and Political Communication in the 2014 Elections to the European Parliament». Electoral Studies, 44, 429-444. http://dx.doi.org/10.1016/j.electstud.2016.04.014

Parmelee, J.H. y Bichard, S.L. (2012). Politics and the Twitter revolution. How tweets influence the relationship between political leaders and the public. Lanham, MD: Lexington Books.

Ramos-Serrano, M., Fernández Gómez, J. y Pineda, A. (2018). «Follow the closing of the campaign on streaming: The use of Twitter by Spanish political parties during 
the 2014 European elections». New Media and Society, 20(1), 122-140. https://doi. org $/ 10.1177 / 1461444816660730$

Risse, T. (2010). A community of Europeans?: Transnational identities and public spheres. Ithaca, NY: Cornell University Press.

Schlesinger, P. (1999). "Changing spaces of political communication: The case of the European Union». Political communication, 16, 263-279. https://doi. org/10.1080/105846099198622

Schmitt, C. (2009) [1932]. El concepto de lo político. Madrid: Alianza.

Schmitt, H., Hobolt, H. y Popa, S.A. (2015). «Does personalization increase turnout? Spitzenkandidaten in the 2014 European Parliament elections». European Union Politics, 16(3), 347-368. https://doi.org/10.1177/1465116515584626

Schulz-Forberg, H. y Strath, B. (2010). The political history of European integration: The hypocrisy of democracy-through-market. Londres y Nueva York: Routledge.

Segado-Boj, F., Díaz-Campo, J. y Lloves, B. (2016). «Objetivos y estrategias de los políticos españoles en Twitter». Index. Comunicación, 6 (1), 77-98.

Seoane Pérez, F. (2013). Political communication in Europe: the cultural and structural limits of European public sphere. Nueva York: Palgrave Macmillan. https://doi. org $/ 10.1057 / 9781137305138$

Thomass, B. (2011). «Deficits and Potentials of the Public Spheres». En J. Trappel (ed.), Media in Europe today (pp. 117-133). Chicago: Intellect.

Tuñón, J. (2017). Comunicación internacional: información y desinformación global en el siglo XXI. Madrid: Fragua.

Tuñón, J., Bouza, L. y Carral, U. (2019). Comunicación europea. ¿A quién doy like para hablar con Europa? Madrid: Editorial Dykinson.

Van Dalen, A., Albaek, E. y De Vreese, C. (2011). «Suspicious minds: Explaining political cynicism among political journalists in Europe». European Journal of Communication, 26(2), 147-162. https://doi.org/10.1177/0267323111404841 